\title{
Preliminary study: Polishing force measurement by viscosity - the return of ketchup polishing
}

\author{
Max Schneckenburger ${ }^{1,2}$, Sven Höfler ${ }^{1,3, *}$, Oliver Fähnle ${ }^{4}$, and Rainer Börret ${ }^{1}$ \\ ${ }^{1}$ Aalen University, Centre for Optical Technologies, Aalen, BW 73430, Germany \\ ${ }^{2}$ Technische Universität Ilmenau, Ilmenau, TH 98693, Germany \\ ${ }^{3}$ Glasgow Caledonian University, Scotland, United Kingdom \\ ${ }^{4}$ Eastern Switzerland University of Applied Sciences, Optical Fabrication Technology, 9471, Switzerland
}

\begin{abstract}
Due to the advantages over conventional polishing strategies, polishing with non-Newtonian fluids are state of the art in precision shape correction of precision optical glass surfaces. The viscosity of such fluids is not constant since it changes as a function of shear rate and time. An example is during the shape correction by polishing with pitch or ice, where pitch flows slowly under its own weight and acts like a solid body during short periods of stress as its viscosity increases. The effect can be measured in the polishing gap with a viscometer. If there is a change in force or a process variation of the polishing pressure in the polishing gap, the viscosity also changes. Conversely, the viscosity value could be used to determine the process variation of the polishing force, at least quantitatively. It is to be expected that the distance of the sensor to the polishing gap (effective zone of the polishing force) and the associated change in the viscosity value has a decisive influence on the accuracy of the measurement resolution. First polishing results will be presented and a bowl feed polishing like approach will be presented
\end{abstract}

Due to the Covid-19 virus and the associated container congestion in Yantian, the construction could not be fully realised at the time of submission. The authors apologise for this. Nevertheless, the theoretical idea is so extensive that the theory and expecta tions are presented below.

\section{Introduction}

The material removal (MRR) in the polish depends primarily on the normal force $\left(\mathrm{F}_{\mathrm{N}}\right)$ and the relative speed $\left(v_{\text {rel }}\right)$, in addition to a number of other process parameters. Preston showed this in his deterministic polishing process and summarised the other process parameters in the Preston-coefficient $\left(\mathrm{c}_{\mathrm{P}}\right)$. Preston defines material removal as follows:

$$
M R R=c_{P} * \frac{F_{N}}{A} * v_{r e l}
$$

This model is accepted in polishing and has been extended by various authors [1].

Currently, the force of the polishing tool can only be measured with a lot of effort:

- If the position of the polishing head is not additionally measured, it is not possible to calculate back to the origin of the force.

- If the polishing tool is not congruent on the sensor $(\Delta)$, lateral tensile and compressive forces $\left(F_{\mathrm{X}}\right)$ are mapped onto the $\mathrm{F}_{\mathrm{Z}}\left(=\mathrm{F}_{\mathrm{X} \text { res Sensor }}\right)$ data. Which makes it impossible to determine the exact $F_{z}$ values. This is shown schematically in Figure 1.

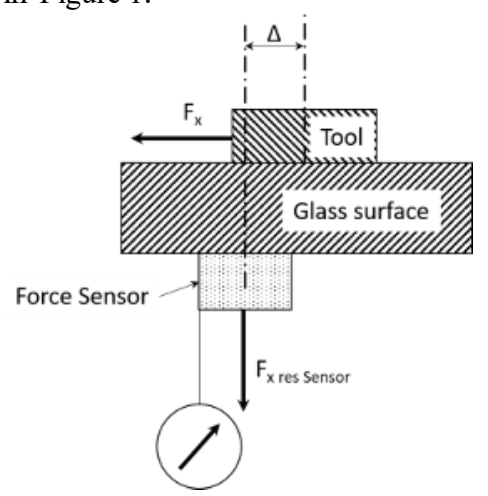

Figure 1: Schematic representation of the effects of the lateral forces on the sensor data

- Sensors directly at the polishing gap have the challenge that the cable is more likely to break due to the movement of the polishing tool and the associated alternating stress on the cable. This particularly affects force sensors directly above the polishing tool.

- The sensor values have drift, which has a visible influence on the measurement data when measured over several hours. According to the manufacturer, the drift can a lways be calculated, but in practice there are considerable measurement uncertainties.

These difficulties concern in particular polishing tools with an axis of rotation parallel to the normal force axis

\footnotetext{
* Corresponding author: sven.hoefler@hs-aalen.de
} 
(eg: eccentric tool). The installation of a force sensor is not a challenge with a polishing wheel. On the other hand, there is the mea surement uncertainty due to vibra tions and the generation of direction-dependent structures by the polishing wheel. In addition, the force sensor is faraway $(\sim 0.1 \mathrm{~m}, 4 \mathrm{inch})$ from the polishing gap.

One possibility of indirect force measurement is measurement above viscosity. In this case, the viscosity of the polishing fluid is measured during the test, which also changes when the force changes. The interesting thing about this is that only the normal force can be measured at a constant relative speed.

Viscosity is the coefficient of internal friction [2]. The internal friction $\left(\mathrm{F}_{\mathrm{R}}\right)$ of a fluid that is to be moved para llel to a flat surface requires force and is equal to the amount of the frictional force. The internal friction force is defined as a function of the contact area (A), the relative velocity between the boundary surfaces (v), the distance between the boundary surfaces (d) and the dynamic viscosity of the fluid $(\eta)$. The dynamic viscosity is the toughness respectively the coefficient of internal friction. This can be applied ana logously to the polishing gap, the area between the polishing tool and the glass workpiece (see Figure 1).

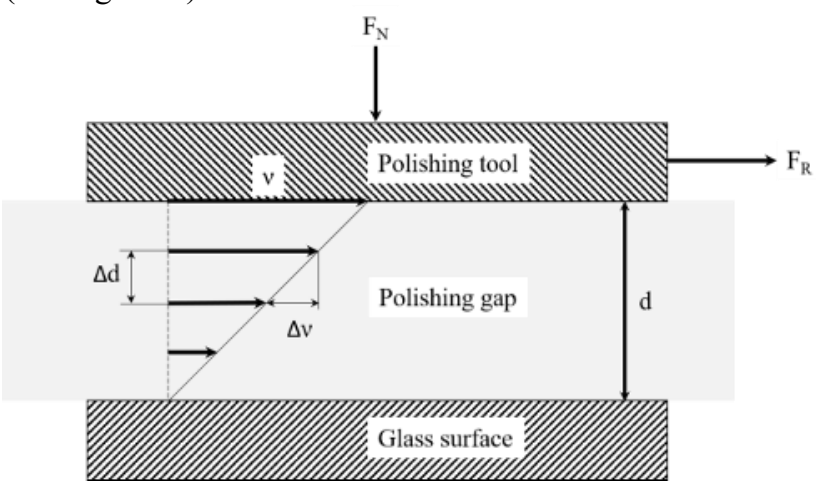

Figure 2: Schematic Polishing gap

$$
F_{R}=\eta * A \frac{\Delta \mathrm{v}}{\Delta \mathrm{d}}
$$

The coefficient of friction $(\mu)$ can be used to relate the viscosity to the normal force:

$$
F_{N}=\mu * F_{R}=\mu * \eta * A \frac{\Delta \mathrm{v}}{\Delta \mathrm{d}}
$$

The following relationship results via the shear rate $(\dot{y})$ and the shear stress $(\tau)$ :

$$
\tau=\eta * \dot{\mathrm{y}}
$$

Formula 2 shows that the viscosity could also be measured indirectly via the polishing gap height instead of mea suring by a specific sensor. HABERLAND et al have already presented a suitable setup for measuring the polishing gap height [3]. This setup has the advantage over the setup presented later that the viscosity can be measured in-situ in the polishing gap.

The dynamic viscosity is strongly temperature-dependent (T), which is why the sensor used has a temperature sensor. $\mathrm{B}$ and $\mathrm{C}$ are empirical constants. However, a lot of frictional heat is generated in the polishing gap, which cannot be fully considered in the following set-up.

$$
\eta=B e^{\frac{C}{T}}
$$

MULLANY ET AL investigated the effects of viscosity in the planarization of wafers [4]. The main focus was the force distribution in the polishing fluid. However, the sensor was not applied in reverse to indirectly measure the force.

The planarization of wafers is very similar to the optical polishing of glasses. Opaline $\left(\mathrm{Ce}_{2} \mathrm{O}\right)$ is usually used as a polishing agent, which is mixed with distilled water. The water serves as a carrier medium for the polishing agent, transports the heat out of the polishing gap and participates in the chemical reaction by dissociating itself. The cerium does not dissolve in water and is present as solid particles. In terms of viscosity, the polishing suspension behaves like a Newtonian fluid and similar to water (see Figure 3).

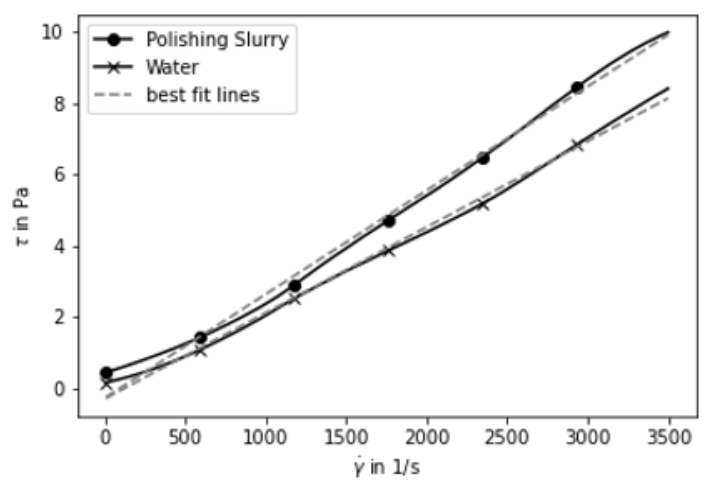

Figure 3: Shows the comparison of the viscosity of water and water with opalines (polishing slurries)

To see the effect of the normal force $F=f(\eta)$ better in the polishing gap and to increase its height, the viscosity of the polishing liquid will be increased. Figure 4 shows the thixotropic behaviour of a pectin-containing liquid (in this case: ketchup). Under increasing load (in this case the shear rate), the polishing slurry liquefies. A liquid with a higher viscosity or a rheopexy liquid could a lso have been used. All these viscosity measurements were made with the Haake RotoVisco laboratory instrument.

Instead of using ketchup as in the previous paper [5], this time $100 \%$ pure apple pectin is used. Currently, an industrial viscosity sensor needs a working range of $>10 \mathrm{~mm}$ because the measuring range has to move in the liquid. Currently, there is no polishing process that has such a polishing gap height, so measurements must be taken close to the polishing gap to avoid dead space. Usually, the polishing gap is less than $1 \mathrm{~mm}$. 


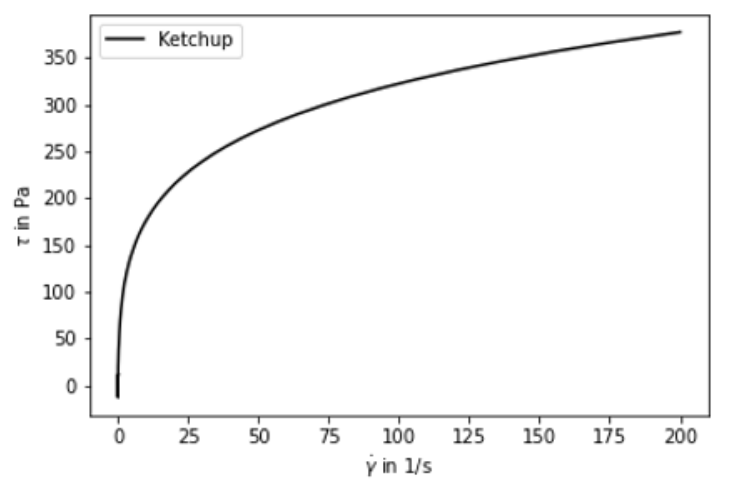

Figure 4: Viscosity measurement of a pectin-containing liquid

\section{Proceeding}

In the following, the experimentalset-up and the planned realisation of the experiment are described.

\subsection{Set up}

Figure 5 shows a schematic of the experimental set-up. The robot polishing head has a rotary motor for the rotation speed of the polishing wheel and a direct drive for applying the force to the glass surface respectively to the polishing gap. It is polished in a polishing bath, i.e. the tool is below the polishing slurry surface. The SOFRASER MIVI sensor is used as the viscosity sensor [6]. Table 1 shows the key data of the sensor. A flat Zerodur lens with a shape deviation of $<1 \mu \mathrm{m}$ is used as the workpiece. To avoid dead space, the sensor is placed close to the polishing gap so that the polishing wheel rotates in this direction. This means that the polishing gap is not measured directly, but at least close to it. Preliminary tests with ketchup showed that the viscosity around the polishing wheel is affected by a force and not only in the immediate area. However, the rotation of the polishing wheel also causes a shearing effect on the process, so that the viscosity is also influenced.

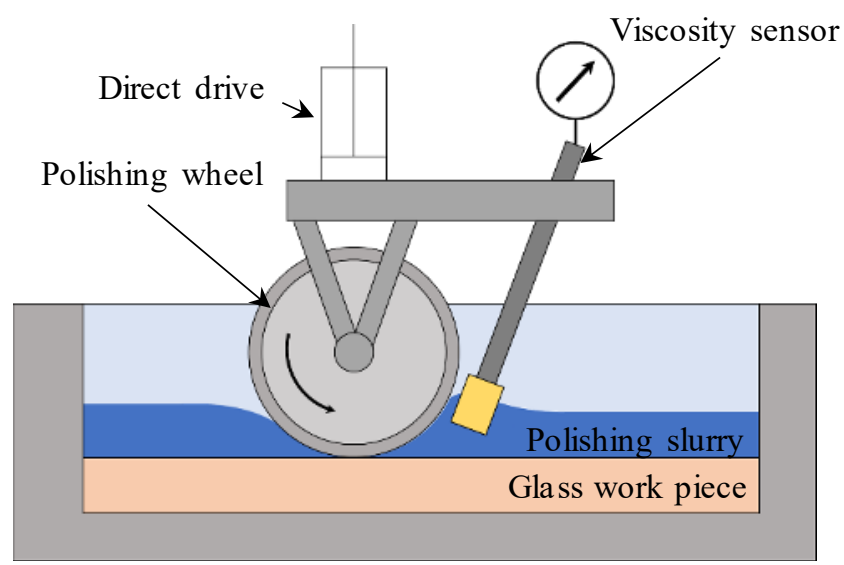

Figure 5: Schematic application of the set up
Table 1. Viscosity sensor key data

\begin{tabular}{|l|r|}
\hline & Viscosity sensor \\
\hline Mea suring range & $100.000 \mathrm{mPas}$ \\
\hline Viscosity precision & $\pm 0,2 \%$ \\
\hline Viscosity accuracy & $\pm 0,5 \%$ \\
\hline Operating temperature & $0 \ldots 200^{\circ} \mathrm{C}$ \\
\hline
\end{tabular}

A load cell above the polishing wheel and a force measuring stage below the glass workpiece are used to check the force applied by the direct drive. Only the force is used as the setting parameter. Varying the output viscosity or the speed will not take place. The speed and some other parameters (including the power of the motor) are monitored and stored during the tests.

\section{Expected results}

The change in force changes the viscosity and normal force can be measured. Despite many influences (including vibrations, process divergences, ...) this is a promising approach. In theory, the decisive factor will be the resolution system of the setup.

\section{Summary and outlook}

In the preliminary study, a setup for mea suring the normal force during the polishing process was presented. At present, no comprehensively satisfactory solutions for force measurement in the polishing process have been found using conventional methods. Therefore, this preliminary study offers a fresh, new, but also unconventionalapproach.

Unfortunately, no tests could be carried out now, but they will be carried out as soon as possible. Currently, only the viscosity sensor is missing.

\section{Acknowledgement}

The work is part of a funded German research project named OpTec 4.0 with the goal of funding young scientist, contract number 13FH003IB6. The authors would like to thank the FederalMinistry of Education and Research for funding this project. 


\section{References}

[1] LuO, Jianfeng; DORNFELD, David: Integrated modeling of chemical mechanical planarization for sub-micron IC fabrication: From particle scale to feature, die and wafer scales; with 14 tables. Berlin : Springer, 2004

[2] KuCHLING, Horst: Taschenbuch der Physik: Mit zahlreichen Tabellen.21., aktua lisierte Aufl., Jub.Ausg. 50 Jahre. München : Fachbuchverl. Leipzig im Carl-Hanser-Verl., 2014

[3] Haberland, R. (Hrsg.); Blume-Werry.U. (Hrsg.); SeyfRIED, P. (Mitarb.); KunZMANn, H. (Mitarb.); MCKeOWN, P. (Mitarb.); WeCK, M. (Mitarb.) : Measurement of Gap and Parameters in the Polishing Process of Optical Glass :

Proceedings of the 6th International Precision Engineering Seminar (IPES 6)/2nd International Conference on Ultraprecision in Manufacturing Engineering (UME 2), May, 1991 Braunschweig, Germany. Progress in Precision Engineering. Berlin, Heidelberg : Springer Berlin Heidelberg, 1991

[4] Mullany, B. ; MainudDin, M.: The influence of process vibrations on precision polishing metrics. In: CIRP Annals 61 (2012), Nr. 1, S. 555-558

[5] SCHNECKENBURGER, Max; SCHIFFNER, Melanie ; BÖRRET, Rainer: High precision glass polishing with ketchup. In: HABERL, Alexander; FÜTTERER, Gerald; FÄHNLE, Oliver W.; RASCHER, Rolf (Hrsg.): Seventh European Seminar on Precision Optics Manufacturing : SPIE, 2020 - 2020, S. 16

[6] SOFRASER: MIVI : Process Viscometer. 2021. URL https://www.sofraser.com/app/uploads/2021/02/FT 3-E-MIVI-134_12-1.pdf 